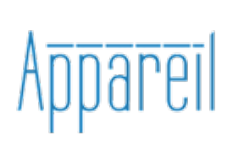

Appareil

$22 \mid 2020$

Une philosophie de l'appareil

\title{
Quand l'art prend soin de vous
}

Les tropismes du care dans l'art aujourd'hui - pour Jean-Louis Déotte

\section{Diane Watteau}

\section{(2) OpenEdition}

Journals

Édition électronique

URL : http://journals.openedition.org/appareil/3542

DOI : 10.4000/appareil.3542

ISSN : 2101-0714

Éditeur

MSH Paris Nord

Référence électronique

Diane Watteau, «Quand l'art prend soin de vous », Appareil [En ligne], 22 | 2020, mis en ligne le 21

décembre 2020, consulté le 26 mars 2021. URL : http://journals.openedition.org/appareil/3542 ; DOI :

https://doi.org/10.4000/appareil.3542

Ce document a été généré automatiquement le 26 mars 2021.

\section{(c)}

Appareil est mis à disposition selon les termes de la Licence Creative Commons Attribution - Pas d'Utilisation Commerciale - Pas de Modification 4.0 International. 


\title{
Quand l'art prend soin de vous
}

\author{
Les tropismes du care dans l'art aujourd'hui - pour Jean-Louis Déotte
}

\author{
Diane Watteau
}

\section{NOTE DE L'AUTEUR}

Ce texte constitue une version reprise et modifiée de l'éditorial du numéro Quand l'art prend soin de vous. Les tropismes du care dans l'art aujourd'hui, publié en avril 2019 dans la revue Plastik. Plus d'un an après la disparition du philosophe Jean-Louis Déotte, il rend hommage à son œuvre grâce à des textes qui témoignent de l'impact de sa pensée sur la philosophie, mais également dans les arts plastiques d'aujourd'hui.

1 Entre éthique et esthétique, art et sciences du vivant, le care - donner et recevoir des soins -, s'affirme comme un concept dans notre société. De nombreux chercheurs et artistes se sont réunis autour de la pensée de Jean-Louis Déotte pour penser la contemporanéité et les conditions de modifications des contextes en intelligence avec le care.

S'il semble encore marginalisé, il nous faut sûrement le penser autrement, comme concept social, moral et politique et le discuter à travers des œuvres dans le cadre de l'économie artistique actuelle.

Selon Christiane Vollaire :

[c]e qui caractérise la notion de care, dès que l'on tente de l'aborder, c'est son caractère parfaitement lisse et sans aspérité. C'est une sorte de galet rond qui glisse entre les doigts et rebondit sur l'eau dans la pratique automatique du ricochet, ne donnant prise à peu près sur rien. Qui contestera en effet qu'il faille prendre soin de soi-même, s'occuper d'autrui, rendre la société vivable et toute cette enfilade de truismes par lesquels on définit « la bonne vie » à grandes lampées de moraline ${ }^{1}$ ?

2 Le care apparait en France comme concept au cours des années 2000. Il est issu du monde protestant anglo-saxon de la fin $\mathrm{du} \mathrm{xx}^{\mathrm{e}}$ siècle, de ses préoccupations sur l'empirisme et les valeurs morales ainsi que de ses polémiques sur la question du genre. Le livre de la philosophe américaine Judith Butler, Trouble dans le genre, paru en 1990, lui sert de terreau féministe. Les chercheurs qui le convoquent, autant en France que 
dans les pays anglo-saxons, sont très majoritairement des femmes, ce qui donne à réfléchir sur le caractère "genré » des pratiques assignées féminines (dans l'espace public et privé) comme objets d'études, mais également sur la théorisation critique même de ces pratiques.

Le care serait-il un concept consensuel ? Comment pourrait-il porter et constituer une pensée novatrice dans des sphères scientifiques, politiques et jusque dans l'art?

Ces questions ont été abordées à l'occasion d'une journée d'études qui accompagnait l'exposition Prendre soin? Celle-ci inaugurait, dans le centre hospitalier de Tonnerre ${ }^{2}$, la nouvelle fonction de la pharmacie hospitalière, devenue espace d'exposition. Ce projet s'inscrivait dans le programme national "Culture et santé». Il réunissait treize artistes : Pierre Baumann, Philippe Bazin, Alain Buffard, Agnès Foiret et Thibault Daelmann, Claude Lévêque, Béatrice Martin, Myriam Mihindou, Françoise Parfait, Ernest Pignon-Ernest, Jeanne Susplugas, Yann Toma, Véronique Verstraete et Diane Watteau.

Prendre soin de qui et pourquoi, quand «le monde va mal» et s'use tant (Jacques Derrida)? La critique de la valeur et des idées reçues sur le soin comme paradigme est peut-être à penser à partir des représentations du corps dans l'art, face aux systèmes politiques et économiques qui l'ont transformé en placement d'un capital, à travers la commercialisation du soin des seniors et des enfants, par exemple. La bienveillance, l'attention et le souci de l'autre suffiraient-ils pour remettre en jeu l'assujettissement de l'homme à la logique marchande actuelle? Représentent-ils vraiment une poche de résistance?

Avant même la crise sanitaire de 2020 et comme une étrange anticipation de celle-ci, l'éthique du care, devenue politique du care - « les politiques de l'ordinaire » comme les appelle Sandra Laugier - faisait déjà l'objet de toute notre attention.

Comment évoquer la manière dont les données médicales deviennent, dans l'art, éléments de fiction? Comment le renouvellement des formes y sera-t-il possible, pour parler des corps fragiles, malades, mortels ? Entre admiration et malaise, comment le corps s'inscrit-il dans l'art en temps de crise majeure, lorsqu'il est entre la vie et la mort, malade, réfugié, exilé ou incarcéré ?

Envisageant tour à tour le corps anatomique, le corps-machine et le corps prophétique, chercheurs plasticiens, philosophes, médecins et anthropologues poursuivent, à travers leurs communications, cette question obsédante : que peut un corps? Ils s'attaquent ici aux masques de l'aliénation actuelle, pour continuer à penser, à la suite de Foucault, un corps «biopolitique»: quelles sont les forces à combattre pour affirmer un "prendre soin »? Quelles violences la focalisation sur le "prendre soin » pourrait-elle viser à occulter?

7 Cette réflexion sur l'art et le care peut se décliner en quatre questions : 1 - Quand le care se mêle d'art. 2 - Quand le care prend le dessus sur l'artistique. 3 - Quand le care devient nécessaire pour penser l'art aujourd'hui. 4 - Quand le care veut faire la leçon à l'art ou Comment « bien penser » dans l'art.

8 La question $d u$ " soin » qui se diffuse dans l'art mérite une réflexion approfondie autour de ses pouvoirs et contre-pouvoirs politiques et artistiques, face à une certaine idéologie dominante du care. La Palme d'or 2017 fut attribuée au film The Square de Ruben Östlund, qui fait de l'appel à l'aide ("Quelqu'un peut m'aider?») une dénonciation d'une logique de la séduction actuelle qui crée un sujet fétichiste 
narcissique $^{3}$ bradant toutes les frontières entre art et réalité et que personne ne viendra ni sauver ni soigner!

«Le monde va mal »! Le care va bien! Comment se révèle la volonté du corps à ne pas se limiter, à devenir expérience ; moyen de connaissance et d'action aujourd'hui dans l'art, en s'ouvrant aux morceaux d'espace qu'il traverse et qui le traversent?

$\mathrm{Si}$ chercheurs et artistes reprennent les questions de l'exclusion ou de la pathologisation des corps dans la représentation, c'est encore pour affirmer que le corps, le sexe et le politique ont plus que jamais partie liée.

En s'appuyant sur Walter Benjamin et sur le concept platonicien de pharmakon, JeanLouis Déotte s'est efforcé de débarrasser l'art de l'exposition «sociologique» des parasites parodiques, des chocs esthétiques... des tics aussi. Un ensemble de questions se posent sur le rapport entre l'exposition, le don et le contre-don, dans une destination anthropologique, donc politique. Or, «ce n'est pas ce qui est dit (le mouvement régressif de l'histoire) qui importe, mais la libération d'une apparition enkystée dans la forme-tableau » (Jean-Louis Déotte).

\section{Quand le care se mêle d'art}

11 Peut-on parler d'une dimension éthique du soin ou même d'une éthique du soin ? Ces questions présupposent que le soin serait une activité comme une autre et laissent entendre qu'il y aurait autant d'éthiques qu'il y a de domaines de l'action humaine. L'article d'Éric Delassus considère le soin comme le cœur même de l'éthique? Il nous est possible d'évaluer l'histoire de la peinture dans un rapport de hantise entre l'éthique et le soin, la bonne santé et la maladie ${ }^{4}$. Que signifierait alors prendre soin de la peinture? Une peinture soignée, avec ce qu'il faut d'ambivalence entre la peinture réparée et réparatrice, se tiendrait à distance de l'extase et du drame pour instaurer des éléments du "vivre ». Agnès Foiret examine les œuvres de Robert Ryman, Roman Opałka, Al Martin et Pierrette Bloch, pour penser la peinture entre le miracle du bien portant et le du pathos du mal portant, pendant du premier ${ }^{5}$. Laurence Gossart observe l'œuvre de Henri Matisse comme un art de guérison, permettant à l'invention d'un rapport au monde, dans la fine articulation entre le cure et le care, de se révéler. Les moments biographiques d'opération, de soin et de création pour Matisse s'initient avec Pierre de Ronsard comme une seconde vie ${ }^{6}$. Il y a du vivant mais il existe d'autres affects, pour Marwan Moujaes. Prenant le deuil à sa charge, l'art serait capable d'agir en désœuvrant le temps et l'espace, mais aussi les corps, le langage, les paysages et les modes d'être de la tristesse. Trouver dans la langue arabe un équivalent au mot care accompagne l'énigme levée des œuvres de Lawrence Abu Hamdan et d'Irina Botea: c'est le mot «ri'aya ». Il signifie aussi "pâturage ", ce qui crée un nouveau rapport entre le care et l'acte de paître ${ }^{7}$.

\section{Quand le care traverse le processus créatif}

La raison de la présence croissante du care, actuellement, incombe à la désaffection interpersonnelle et environnementale. L'observation de Paul Ardenne des œuvres est traversée par les questions d'harmonie collective ou écologique (Mierle Laderman Ukeles, Tattfoo Tan, Vaughn Bell, etc.). Cet art altruiste, par choix, 
est citoyen par essence et par développement: du côté de la collectivité et contre la privatisation du monde. Une correction, une rectification du point de vue et des comportements nécessaires dans le sens de l'humanisme, de la générosité, du sens du collectif, " du désir de ne pas crever sans réagir ni sans se faire force de proposition », innerve l'art sous sa forme curatrice, d'essence éco-éthique, loin des musées et des cimetières institutionnels. Cette création altruiste, mise d'emblée au service d'autrui, dans la lignée conceptuelle de l'" autrisme ", cher naguère à un artiste tel que Robert Filliou, prend tout son sens aujourd'hui ${ }^{8}$. Faire $d u$ bien n'est pas qu'un adage. Les séries télévisées prennent vraiment soin de nous pour Sandra Laugier. Elle montre le décalage frappant entre l'influence intellectuelle, politique et morale des séries, leur place dans les conversations et la vie ordinaire et la prise en compte de cette réalité dans la recherche. L'ambition des séries télévisées transforme non seulement nos visions du monde, mais également le monde même, en suscitant, en exerçant et en représentant le care, non plus sur le modèle classique et éculé de l'identification et de la reconnaissance, mais de la fréquentation et de la familiarisation, voire de l'affection ${ }^{9}$. Les affects traversent la performance également, l'artiste Anatoli Vlassov utilise le concept artistique "tenser", misant sur une dialectique de la relation à l'altérité. L'autisme y est pris à l'envers du soin. C'est-à-dire non pas comme une maladie mais comme une rencontre. Il ne s'agit pas de soigner, mais de prendre le risque de créer ensemble ${ }^{10}$. "L'art lave notre âme de la poussière du quotidien. " Cette phrase de Pablo Picasso engage la réflexion de Sylvie Dallet : par l'usage des onguents et des eaux, l'artiste prend place parmi les guérisseurs, détournant le médicament par la transe interprétative qui l'accompagne. Les expériences artistiques d'Hervé Fischer, Marion Baruch, Myriam Mihindou et Lionel Sabatté s'inspirent des rituels énergétiques dans une créativité curatrice ${ }^{11}$.

\section{Quand le care fait corps avec l'art aujourd'hui}

13 Le processus de création de l'artiste Céline Martin s'articule intimement à son travail d'éducatrice spécialisée. Viktoria von der Brüggen étudie ses gestes et ses matériaux privilégiés, révélateurs des thèmes explorés par l'artiste dans la sollicitude envers l'autre. Toucher, étreindre, caresser, réparer, mais également explorer les qualités physiques des matériaux, notamment de la terre et du latex, déterminent une démarche créative fondée sur l'expériences des corps en handicap ou non ${ }^{12}$. Marion Laval-Jeantet analyse le processus d'appropriation de la maladie dans la vie et l'œuvre des artistes Joseph Beuys, Anna Halprin et Salvatore Iaconesi, à l'image de LaCura, dans d'autres perspectives thérapeutiques "pour une pensée vivante et opérationnelle » ? L'" artiste-médecin » parle de sa guérison et des rituels de guérison ${ }^{13}$. Prendre soin nous met au défi, aujourd'hui, de réinventer des formes esthétiques et des gestes artistiques dans une expérience esthétique concrète et sensible : Corps commun de l'artiste Sarah Roshem est un ensemble d'œuvres qui permet de vivre une expérience dans laquelle nos corps sont reliés à deux, à quatre ou à plus pour distinguer ce qui distingue l'ensemble du commun ${ }^{14}$. 


\section{Quand le care veut faire la leçon à l'art ou Comment « bien penser » dans l'art avec le care}

14 L'idéologie du care peut reproduire un discours religieux de l'universel et de la compassion qui pose un déni sur l'effectivité du désir de violence comme dynamique vitale. Christiane Vollaire nous alerte à propos de ce déni dans l'histoire même des systèmes sociaux. Plutôt que penser la sollicitude comme élément moteur du care, on pourra choisir la sollicitation, qui pousse à susciter en l'autre sa puissance plutôt que sa faiblesse. À force de multitude, les images considérées comme fléau social peuvent être l'objet de soin par certains artistes ${ }^{15}$ (Adam Broomberg et Oliver Chanarin, Alfredo Jaar, Taryn Simon et Thomas Hirschhorn). Dans la superposition des questions du care et du scopique, Anna-Maria Le Bris montre que l'acte de prendre soin peut être une alternative à cette crise scopique : comment voir ensemble trop d'images ${ }^{16}$ ? Le monde des curios serait-il une réponse possible ? Florian Gaité analyse ce monde merveilleux proche des rêveries de Roger Caillois devant les paésines dites "pierres-à-images ", mais aussi les collections de minéraux investissent désormais régulièrement les expositions d'art contemporain. La question de la curiosité à l'âge de l'anthropocène restaurerait-elle une relation de soin entre les hommes et leur environnement ? Dans la contemplation des curios se réalise ainsi la synthèse des dimensions cognitive et affective de l'attention, à la fois scrupule méthodique et soin curatif. Nous voilà dans l'ère des néo-curios ${ }^{17}$ (avec Hugues Reip, Jimmie Durham, Kelly Jazvac et Gabriel Orozco). La curiosité esthétique s'est affirmée comme un moyen de remédiation, au double sens d'apporter un remède (soigner un état critique) et de restaurer une médiation (réparer le lien de l'homme à la nature). L'hypothèse du pharmakon, du fait que le rapport à l'autre, quand il est médiatisé par l'hospitalité, concerne un certain type d'écriture et d'inscription, est l'objet de l'étude d'Adolfo Vera. L'œuvre s'avère être une mise en mémoire développée par Jacques Derrida, impliquant une politique de la spectralité qui obligera à la pensée à construire ses concepts au-delà de toute identité. Prendre soin de ses fantômes deviendrait une nouvelle activité ${ }^{18}$ ? De la spectralité à la "pensivité ", la philosophe Fabienne Brugère rapproche l'art et l'éthique du care pour réfléchir la conception des images du côté de la pensée. Une théorie de la vérité en art, d'inspiration heideggérienne, peut rendre classiquement raison d'un "prendre soin » des œuvres d'art. Qu'en est-il alors d'une éthique du care adressée à l'art ? Sophie Calle demeure l'exemple idéal ${ }^{19}$.

\section{Conclusion}

Prendre soin du patrimoine est l'objet du texte qui conclut pour un temps les expériences multiples du care avec l'art, dans l'art et pour l'art enregistrées dans le numéro 6 de Plastik. La tentation de la permanence restée intacte dans l'art se voit bouleversée par ce concept du care. La tragédie grecque, nous le rappelle JeanLouis Déotte, était en Grèce une performance et non un genre littéraire. C'était plutôt, même, une performance rituelle, un spectacle choral offert à Dionysos. Penser la tragédie aujourd'hui, c'est penser le passage de l'ère de la reproduction à l'aura du marbre des temples antiques (à travers les œuvres de Daniel Buren et de Yann Kersalé mais également dans l'événement Monumenta). L'art désire sa part d'impermanence dans des expériences furtives, comme si la maïeutique restait le dernier mot de 
l'esthétique. La pensée du philosophe Jean-Louis Déotte continue sa course vers une forme chorale, une énonciation particulière avec un « je » ou un « nous » incarnés dans des œuvres hospitalières ${ }^{20}$.

Figure 1. Martine et Jean-Louis Déotte
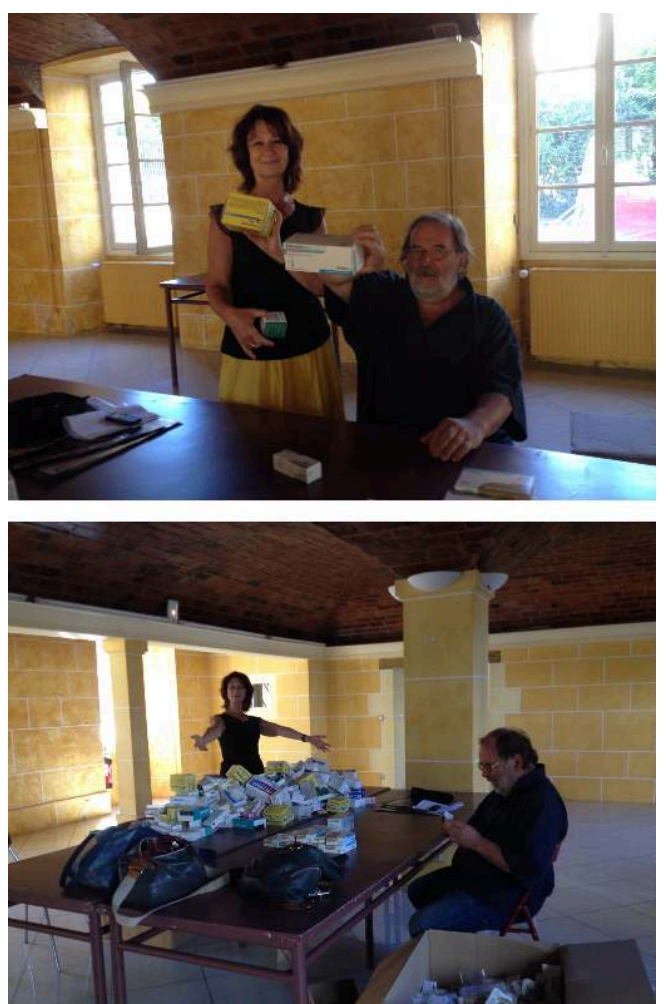

Note: Préparation de l'exposition Prendre soin ? 4 juillet 2017, centre hospitalier de Tonnerre exposition Prendre soin ?, co-commissariat Diane Watteau et Jean-Louis Déotte, 2017

Photographie @ Diane Watteau 
Figure 2. Mass destruction

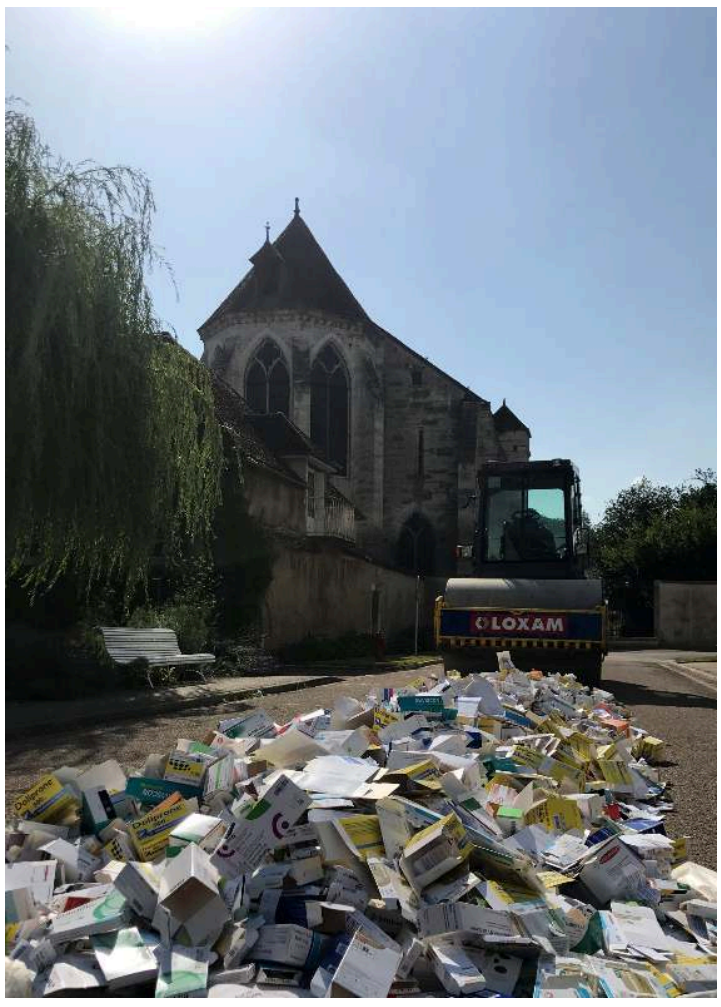

Note : Jeanne Susplugas, Mass destruction, installation/performance, rouleau compresseur, boîtes de médicaments, dimensions variables, exposition Prendre soin ?, pharmacie du Centre hospitalier du Tonnerois, Tonnerre, 4 juillet 2017

Photographie () Jeanne Susplugas 
Figure 3. Installation in situ

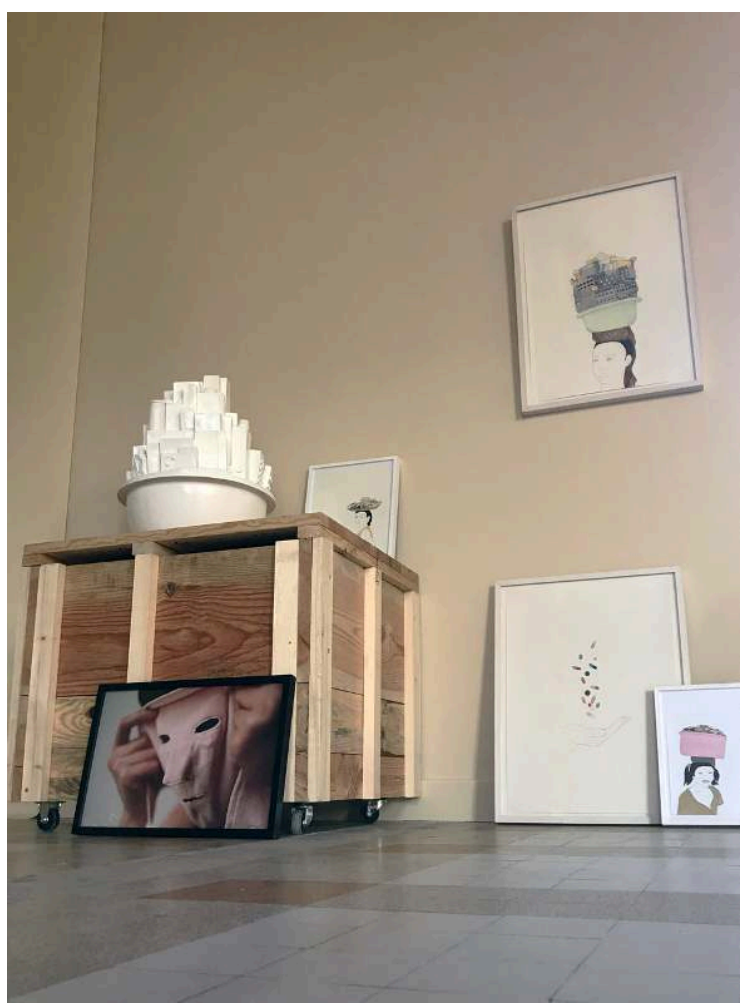

Note : Au premier plan : Mask, c-print, 40×60 cm, 2009 ; sur la caisse, Pharmacie gazon, résine, 2009 ; mur et sol : Sale's woman in Bamako, $59.4 \times 42 \mathrm{~cm}$ - Sale's woman in Lagos, $32 \times 24 \mathrm{~cm}$ - Sale's woman in Djenne, 27,9x19,2 cm - Pills hand, 59,4×42 cm, 2008

Photographie @ Jeanne Susplugas

\section{NOTES}

1. Christiane Vollaire, «Prendre soin : contre qui ? ", Appareil, Varia, Articles, 2016. URL : http:// journals.openedition.org/appareil/2346 [consulté le 02/07/2020]

2. Jean-Louis Déotte, "L'exposition "Prendre soin » comme déniaisement ", Appareil, Varia, Galerie, 2018. URL : https://journals.openedition.org/appareil/2641 [consulté le 17/08/2020]

3. Anselm Jappe, La société autophage. Capitalisme, démesure et autodestruction, Paris, La Découverte, 2017.

4. Éric Delassus, "Le soin ou l'éthique en acte ", Plastik, nº 6, 2019. URL : https://plastik.univparis1.fr/le-soin-ou-lethique-en-acte/

5. Agnès Foiret, « La peinture soignée ", Plastik, $n^{\circ}$ 6, 2019. URL : https://plastik.univ-paris1.fr/lapeinture-soignee/

6. Laurence Gossart, «Henri Matisse l'art d'une guérison, l'invention d'un rapport au monde », Plastik, $\quad \mathrm{n}^{\circ} 6, \quad 2019$. URL: https://plastik.univ-paris1.fr/henri-matisse-lart-dune-guerisonlinvention-dun-rapport-au-monde/

7. Marwan Moujaes, «Habiter les pâturages oubliés", Plastik, $\mathrm{n}^{\circ} 6,2019$. URL : https:// plastik.univ-paris1.fr/habiter-les-paturages-oublies/ 
8. Paul Ardenne, «De quelques pratiques du "care" (le "soin", l'“éthique de la sollicitude") dans l'art du tournant du 21e siècle", Plastik, $\mathrm{n}^{\circ}$ 6, 2019. URL: https://plastik.univ-paris1.fr/dequelques-pratiques-du-care-le-soin-lethique-de-la-sollicitude-dans-lart-du-tournant-du-21esiecle/

9. Sandra Laugier, «Comment les séries TV prennent soin de nous », Plastik, $n^{\circ} 6,2019$. URL : https://plastik.univ-paris1.fr/comment-les-series-tv-prennent-soin-de-nous/

10. Anatoli Vlassov, «TENSER l'autisme à l'envers du soin. CARE comme rencontrer et non inclure », Plastik, $\mathrm{n}^{\circ}$ 6, 2019. URL : https://plastik.univ-paris1.fr/tenser-lautisme-a-lenvers-dusoin-care-comme-rencontrer-et-non-inclure/

11. Sylvie Dallet, «L'eau de l'art », Plastik, $n^{\circ} 6$, 2019. URL : https://plastik.univ-paris1.fr/leau-delart/

12. Viktoria von der Brüggen, «Toucher l'humain : Gestes de sollicitude dans le processus créatif de Céline Martin », Plastik, $\mathrm{n}^{\circ}$ 6, 2019. URL: https://plastik.univ-paris1.fr/toucher-lhumaingestes-de-sollicitude-dans-le-processus-creatif-de-celine-martin/

13. Marion Laval-Jeantet, "L'artiste face à la maladie. De Josef Beuys, Antoni Tàpies et Sam Francis... à Salvatore Iaconesi ", Plastik, $\mathrm{n}^{\circ}$ 6, 2019. URL : https://plastik.univ-paris1.fr/ lartiste-face-a-la-maladie-de-josef-beuys-antoni-tapies-et-sam-francis-a-salvatore-iaconesi/

14. Sarah Roshem, «Corps Communs : une expérience esthétique du prendre soin », Plastik, $\mathrm{n}^{\circ} 6$, 2019. URL : https://plastik.univ-paris1.fr/corps-communs-une-experience-esthetique-duprendre-soin/

15. Christiane Vollaire, «Prendre soin: contre qui ?», Appareil, Varia, Articles, 2016. URL : http://journals.openedition.org/appareil/2346 [consulté le 02/07/2020]

16. Anna-Maria Le Bris, « Du soin des images à l'autonomie du regard », Plastik, $n^{\circ} 6,2019$. URL : https://plastik.univ-paris1.fr/du-soin-des-images-a-lautonomie-du-regard/

17. Florian Gaité, "Quand la curiosité (re)devient soin. Les curios dans l'art contemporain », Plastik, $\mathrm{n}^{\circ}$ 6, 2019. URL : https://plastik.univ-paris1.fr/quand-la-curiosite-redevient-soin-lescurios-dans-lart-contemporain/

18. Adolfo Vera, «Prendre soin des fantômes : le pharmakon artistique », Plastik, $n^{\circ}$ 6, 2019. URL : https://plastik.univ-paris1.fr/prendre-soin-des-fantomes-le-pharmakon-artistique/

19. Fabienne Brugère, "Prenons soin" des œuvres. Comment aller de Heidegger à Sophie Calle ? », Plastik, n 6, 2019. URL : https://plastik.univ-paris1.fr/prenons-soin-des-oeuvrescomment-aller-de-heidegger-a-sophie-calle/

20. Jean-Louis Déotte, «Prendre soin du patrimoine», Plastik, $\mathrm{n}^{\circ} 6$, 2019. URL: https:// plastik.univ-paris1.fr/prendre-soin-du-patrimoine/

\section{RÉSUMÉS}

En hommage à Jean-Louis Déotte, Diane Watteau rappelle la fécondité de ses réflexions sur l'art et le care, notamment dans l'analyse par le philosophe d'une exposition organisée à la Pharmacie, un espace pour l'art contemporain situé dans le pavillon Dormois de l'hôpital de Tonnerre à l'été 2017. L'éditorial du $n^{\circ} 6$ de la revue Plastik lui avait été dédié. Il rendait hommage à son œuvre grâce à des textes qui témoignent de l'impact de sa pensée sur la philosophie, mais également dans les arts plastiques d'aujourd'hui. Les analyses principales de cette lecture sont ici reprises. 
INDEX

Mots-clés : care, art contemporain, patrimoine, éthique en acte, sollicitude

\section{AUTEUR}

\section{DIANE WATTEAU}

Artiste, maître de conférences en arts plastiques à l'École des Arts de la Sorbonne, Institut ACTE (EA7539-Paris1), critique d'art (Association internationale des critiques d'art), commissaire indépendante, adjointe à la rédaction de Savoirs et Clinique, Revue de Psychanalyse ;

diane.watteau@univ-paris1.fr 